\title{
Comparison of Noise Reduction Methods in Photoacoustic Microscopy
}

\author{
Gokhan Guney ${ }^{1 *}$, Nasire Uluc ${ }^{2}$, Aytac Demirkiran $^{2}$, Esra Aytac-Kipergil ${ }^{2}$, \\ Mehmet Burcin Unlu ${ }^{2}$, Ozlem Birgul ${ }^{1}$
}

\author{
${ }^{1}$ Department of Biomedical Engineering, Ankara University, 06830, Ankara, Turkey \\ ${ }^{2}$ Department of Physics, Bogazici University, 34342, Istanbul, Turkey.
}

\author{
Corresponding author: Gokhan Guney* \\ Mailing address: Ankara University, Gölbası 50. Year Campus, Department of \\ Biomedical Engineering, Gölbası, Ankara, 06830, Turkey \\ Tel.: +90-312-600 $0100-1551$ \\ E-mail: gguney@ankara.edu.tr
}

\begin{abstract}
Photoacoustic microscopy (PAM) is classified as a hybrid imaging technique based on the photoacoustic effect and has been frequently studied in recent years. Photoacoustic (PA) signals are inherently recorded in a noisy environment and are also exposed to noise by system components. Therefore, it is essential to reduce the noise in PA signals to reconstruct images with less error. In this study, an image reconstruction algorithm for PAM system was implemented and different filtering approaches for denoising were compared. Studies were carried out in three steps: simulation, experimental phantom and blood cell studies. FIR low-pass and band-pass filters and Discrete Wavelet Transform (DWT) based filters (mother wavelets: "bior3.5", "bior3.7", "sym7") with four different thresholding techniques were examined. For the evaluation purpose, Root Mean Square Error (RMSE), Signal to Noise Ratio (SNR) and Contrast to Noise Ratio
\end{abstract}


(CNR) metrics were calculated. In the simulation studies, the most effective methods were obtained as; sym7/heursure/hard thresh. combination (low and medium level noise) and bior3.7/sqtwolog/soft thresh. combination (high-level noise). In experimental phantom studies, noise was classified into five levels. Different filtering approaches perform better depending on the SNR of PA images. For the blood cell study, based on the standard deviation in the background, sym7/sqtwolog/soft thresh. combination provided the best improvement and this result supported the experimental phantom results.

Keywords Photoacoustic (PA), Photoacoustic Microscopy (PAM), Discrete wavelet transform (DWT), Signal-to-noise ratio (SNR), Signal denoising

\section{INTRODUCTION}

Photoacoustic imaging (PAI) is a new and emerging method, based on the measurement of ultrasonic acoustic waves produced by thermoelastic expansion. The generation of photoacoustic effect begins with the conversion of the electromagnetic radiation energy to heat by the tissue which is stimulated by short-pulsed laser beams. After the energy is converted to heat, the tissue temperature rises. The rising temperature causes the thermal expansion in the tissue and acoustic pressure occurs [1], [2]. To generate acoustic pressure, nonionizing waves such as short laser pulses or $\mathrm{rf}$ pulses are mostly used [3]

In the last decade, PAI studies and development of the PAI systems have shown significant progress. Several studies have been conducted in the last decade, both in system development and application fields. A few examples can be briefly mentioned as 
follows. In 2008, Kolkman et al. [4] conducted a PAI study to follow the growth of pancreatic tumor cells in a rat. In 2011, Jansen et al. [5] demonstrated a study on the intravascular photoacoustic imaging of human coronary atherosclerotic plaque using a custom build catheter. In 2013, Papadopoulos et al. [6] presented an optical-resolution photoacoustic imaging system, which uses a multimode fiber as the source of the optical excitation field and in 2018, Allen et al. [7] demonstrated a laser scanning optical resolution photoacoustic microscopy system based on a stationary fiber optic sensor. Combining high ultrasonic resolution and optical contrast, PAI is able to present deep imaging with high resolution beyond the optical diffusion limit using the low scattering feature of the ultrasonic waves in biological tissues [8]. Photoacoustic (PA) signals are nonlinear and non-stationary signals and are affected by noises which are produced by different sources such as, thermal acoustic noise from the medium, thermal noise from the ultrasonic transducer or electromagnetic (EM) interference [9],[10]. Noise in PA imaging has specific features. For example, while the thermal acoustic noise has a random distribution which can be modeled as Gaussian noise [11], noise arises from the ultrasonic transducer can be modeled as Johnson noise [12]. The fixed pattern noise produced by EM interference also has its own noise characteristics [11].

To reduce the thermal noise in the medium, one approach can be to cool the environment. This attempt, however, brings a tradeoff between noise and PA signal amplitude [9]. Some signal processing methods to enhance PA signals have been presented in the literature. For reducing the random noise, averaging is commonly used. This approach is based on the use of multiple data, therefore, it is time-consuming and not useful for moving targets. Another conventional method for denoising the random 
noise is filtering. Usually spectrum of the PA signals and the noise overlap. For this reason, filtering a specific frequency band may not be an effective method due to potential discarding of useful signal components when there is noise overlapping with the frequency band of PA signals [3]. Recently, wavelet transform based filtering has become an effective denoising method. The transform decomposes the signals into a series of basis functions with different coefficients and the small values of the coefficients which correspond to the noise can be removed using thresholding [13].

In the biomedical field, reducing noise using wavelet transform in biological signals such as Electrocardiography (ECG) [14], [15] and Electromyography EMG [16], [17] is frequently studied. There are, however, a few studies in the relatively new field of photoacoustic imaging using wavelet-based denoising [18]. In this paper, we report the results of our comparative analysis of different wavelet basis functions and FIR lowpass and band-pass filters for PA signal denoising.

\section{MATERIALS and METHODS}

In this section, PA signal generation mechanism, experimental setup, filtering methods, image reconstruction algorithm, and evaluation metrics are given in detail.

\subsection{Photoacoustic Signal Generation}

The basic principle of PA effect and signal formation can simply be explained as follows: The tissue absorbs the EM radiation energy, the absorbed energy is converted to heat, and the temperature increases. As the temperature increases, the tissue undergoes thermal expansion and an acoustic pressure is generated. Formation of the 
acoustic signal depends on parameters such as laser pulse duration, energy, wavelength etc. Theoretically, the acoustic signal can be defined by the acoustic wave equation (1).

$\nabla^{2} p(r, t)-\frac{1}{V_{s}^{2}} \frac{\partial^{2}}{\partial t^{2}} p(r, t)=-\frac{\beta}{C_{p}} \frac{\partial}{\partial t} H(r, t)$

where, $p(r, t)$ is the acoustic pressure at a specific location $r$ and time $t . H(r, t)$ is the heating function, defined as transformed thermal energy in spatial position $r$ and at time $\mathrm{t}$; $\mathrm{Cp}$ is the isobaric specific heat $(\mathrm{J}) ; \beta$ is the thermal volume expansion coefficient $(\mathrm{K}$ 1) and Vs is the speed of sound [1]. The solution of the equation for a Gaussian source is given in [19].

\subsection{Data Acquisition System}

In this study, the PAM system developed by Boğaziçi University Medical and Biophysics Research Group Laboratory was used. The main blocks of the system are shown in Fig. 1. In the system, process starts with the internal trigger signal from the laser to trigger the data acquisition. The light rays emitted from the laser are passed through a filter selected according to the optical absorption feature of the tissue or object, and after collimation, the beam reaches the mirror. The reflected beam focuses on the area to be imaged with the microscope objective and the acoustic pressure signals generated by the thermal expansion of the tissue are recorded by an ultrasonic transducer. These recorded signals are pre-amplified and then transmitted to the receiver [20]. A typical PA signal acquired by the PAM system and parts of the signal are given in Fig. 2.

\subsection{Image Reconstruction Algorithm}


The algorithm developed for image reconstruction follows the steps given in Fig 3. The trigger signal is a high amplitude and information-free signal recorded by the transducer during laser triggering. At the correlation calculation step, oscillations in the triggering signal may affect and mislead the results. To eliminate the effect of triggering signal in the algorithm, first the spectrograms were generated using Short Time Fourier Transform (STFT) in the MATLAB ${ }^{\circ}$; then, an integral calculation is made on the spectrograms for each pixel and the values over the threshold value were masked. After masking the triggering signals, simulated acoustic signal is generated and correlation for each pixel is calculated. Then, the maximum correlation interval was found and the location of the PA signal was determined.

In the next step of algorithm, the filtering process was applied to the signals or directly passed to the last step to generate the original image. In the filtering step, different filters were used to denoise the PA signal. In this step, FIR low-pass and band-pass filters and DWT based filters using four different threshold selection rules were used. In the last step, using trigger signal masked and filtered signals or merely trigger signal masked raw signals, images were generated.

\subsection{Photoacoustic Signal Denoising}

MATLAB ${ }^{\circledR}$ Signal Processing Toolbox and Wavelet Toolbox functions were used to implement noise reduction step. In this section, FIR filters and Wavelet filters used for denoising are explained. 


\subsubsection{FIR filter based denoising}

The filters produced with the FIR1 function in the MATLAB ${ }^{\circledR}$ Signal Processing Toolbox uses window-based filtering. This function uses the least squares approach to compute the filter coefficients at the filter rank specified for the selected window function. The FIR filters in the study were designed using a Hamming window. An ultrasonic transducer with a center frequency of $3.5 \mathrm{MHz}$ was used in the PAM system. Considering the center frequency of the ultrasonic transducer, the cut-off frequency for the low-pass filter was set to $5 \mathrm{MHz}$ and the cut-off frequencies for the band-pass filter were set to 1.2 to $5 \mathrm{MHz}$

\subsubsection{Discrete wavelet transform based denoising}

In discrete wavelet transform denoising, first a suitable mother wavelet must be selected and then decomposition, thresholding and reconstruction steps are performed. Mother wavelet selection is the most critical step and depends on the wavelet characteristics or the similarity between the signal and mother wavelet [21]. For this study, bior3.5, bior3.7 and sym7 wavelets in the MATLAB® wavelet toolbox were selected as the mother wavelets, considering their similarity to the PA signal. The decomposition step is carried out by selecting the appropriate degree of decomposition. In decomposition steps, low-pass and high-pass filters are used based on the characteristics of the mother wavelet. The output of these filters respectively called as approximation and detail coefficients. Depending on decomposition level filters are applied to the detail coefficients at each step. In the process of denoising, the next step is thresholding. Thresholding is a signal estimation technique that uses the properties of the wavelet transform [22]. Traditional thresholding methods are soft and hard thresholding proposed by Donoho and Johnstone, [23]. In hard thresholding, the wavelet coefficients 
smaller than the threshold value is set to zero and higher values than the threshold stay unaltered. In the soft thresholding method, if the absolute value of the wavelet coefficients are less than or equal to the threshold value, then the coefficients are set to zero. If the absolute value is greater than the threshold value, the coefficients are shrunk towards the zero. Hard and soft thresholding equations expressed as follows:

$$
\begin{gathered}
\mathrm{x}_{\text {hard }} \\
=\left\{\begin{array}{cc}
\mathrm{x}, & |\mathrm{x}|>\text { threshold } \\
0, & |\mathrm{x}|<\text { threshold }
\end{array}\right. \\
\mathrm{x}_{\text {soft }} \\
=\left\{\begin{array}{cc}
\operatorname{sign}(\mathrm{x})(|\mathrm{x}|-\text { threshold }), & |\mathrm{x}|>\text { threshold } \\
0, & |\mathrm{x}|<\text { threshold }
\end{array}\right.
\end{gathered}
$$

There are different threshold selection rules in MATLAB® Wavelet Toolbox and four of the commonly used are selected for this study. These are:

- Rigrsure: Rigrsure thresholding uses the principle of Stein's unbiased risk estimate (SURE) to select the threshold.

- Sqtwolog: The threshold value is proportional to the noise $\sigma$ (estimated from data) and $\mathrm{N}$ the number of samples $\sigma \sqrt{2 \log (N)}$.

- Heursure: Heursure thresholding is a combination of Rigrsure and global (sqtwolog) thresholding method. 
- Minimaxi: Minimaxi selection rule uses a fixed threshold to obtain the minimum error in root mean square error calculation.

The last step for the denoising is reconstruction. The filtered signal is reconstructed using unchanged approximation coefficients and thresholded detail coefficients.

\subsection{Performance Evaluation Metrics}

For the performance evaluation of filtering methods different metrics were used in the study. In the literature, Root Mean Square Error (RMSE) is frequently used for the performance analysis when the original signal is known. In this study, RMSE calculation was used to evaluate the performance of the filters at the simulation study. The formula of the RMSE expressed as follows:

$$
\operatorname{RMSE}=\sqrt{\frac{1}{\mathrm{~N}}\left(\mathrm{x}_{\mathrm{i}}-\mathrm{x}_{\mathrm{i}}^{\prime}\right)}
$$

Where $x i$ is $i$. element of the original signal $x$ and $x ' i$ is the $i$. element of the denoised signal x'i and $\mathrm{N}$ is the number of signal samples.

PA signals acquired from the PAM system inherently contain noise and calculation of the RMSE becomes meaningless. In the phantom study step, images of phantom material, generated using denoised signals and raw signals were used. Signal-to-Noise Ratio (SNR) and Contrast-to-Noise Ratio (CNR) metrics were calculated on the images for the evaluating of filter performance. The formula of the metrics expressed as follows: 


$$
\begin{aligned}
& \text { SNR }=\frac{\text { mean }(\text { object })}{\text { std(background })} \\
& \text { CNR }=\frac{2(\mid \text { mean }(\text { object })-\text { mean }(\text { background }) \mid)}{(\text { std(object })+\text { std }(\text { background }))}
\end{aligned}
$$

Where 'std' is standard deviation, 'mean' is mean value, 'background' and 'object' are selected region of interest on the reconstructed image.

\section{RESULTS}

This section includes the results of the PA signal denoising process. In the study, filtering studies were carried out in three steps. In the first step, the synthetic noise was added to simulated time signals and SNR improvement was observed in the controlled environment. RMSE calculated between filtered signal and the actual signal (analytically calculated) was used for SNR improvement evaluation. Then the algorithms were applied on experimental phantom signals. For these cases, the original signal is not known and therefore noise improvement cannot be calculated using RMSE value. Therefore, the improvement was evaluated using SNR and CNR values calculated from reconstructed images, before and after filtering. Finally, the data recorded from the blood cell experiment were used and results are presented in subsequent sections.

\subsection{Simulation Results}

First, simulated time signal was generated using the analytical solution of wave equation for a Gaussian source in [19] using $\tau=5 \mathrm{~ns}, \sigma=8 \mu \mathrm{m}, \mathrm{R}=8 \mu \mathrm{m}, \mathrm{r}=16 \mu \mathrm{m}$ (calculated as $\mathrm{r}=2 \mathrm{R}$ ) and Vst $=1520 \mathrm{~m} / \mathrm{sec}$. (acoustic velocity in the blood). Then, Gaussian synthetic noise with amplitudes 30\%, (low level noise) 50\% (medium level noise) and 100\% (high-level noise) of the peak value of the generated signal was added 
to the original signal. Noisy signals were filtered with Hamming window-based FIR low-pass (LPF), band-pass (BPF) filters and DWT based filters, and then RMSE were calculated to evaluate the filter performance. Table 1 shows the results for LPF, BPF and three different mother wavelets with four different thresholding rules using hard or soft thresholding. Fig. 4 shows the simulated signal, noisy signals, and filtered signal examples.

In Table 1, the most effective method was obtained to be (sym7/heursure/hard thresh.) combination for reducing the low and medium level Gaussian noise cases. This combination reduces the RMSE from 0.30 to 0.11 for low-level noise and reduces the RMSE 0.50 to 0.17 for medium level noise. The highest improvement for high-level noise is obtained by using the (bior3.7/sqtwolog/soft thresh.) wavelet case, which reduces the RMSE from 1.00 to 0.30 . At this noise level performance of LPF and BPF was worse compared to low/medium noise cases.

\subsection{Experimental Phantom Results}

In this section, the USAF Resolution Test Target (Fig. 5) and the system explained in chapter 2.2 was used for data acquisition. Test target was immersed in water and then scanned with PAM system [20]. Experiments with various wavelength and laser power parameters were carried out. For all experiments, $65 \mathrm{kHz}$ pulse repetition rate was used. Table 2 provides the list of different cases. In I1 and I 2 cases, 2nd element in the 5 th group, and for I3-I11 cases 6th element in the same group were used. For Element 2, the line thicknesses are $13.92 \mu \mathrm{m}$ and for element 5, the line thicknesses are $8.77 \mu \mathrm{m}$. Fig. 5 shows the test material. 
Acquired signals were filtered using LPF, BPF and DWT based filters and images of the phantom were generated. To evaluate the filter performance, SNR and CNR metrics were calculated on reconstructed images. In some imaging studies, three different methods gave the similar results. For comparative analysis, Table 3 shows both the initial SNR and CNR values of the images and the result of the best of the three methods, where the improvement is expressed in terms of percentage noise reduction with respect to initial value.

In table 3 , it is seen that, three different filtering methods dominate the results. In some cases, however, other filtering methods gave similar results in general. For I3 case the highest SNR improvement was achieved as $142.55 \%$ and the highest CNR improvement was achieved as $300.75 \%$ when sym7/minimaxi/soft thresh. combination is used. For the same case, bior3.7/rigrsure/soft thresh. and sym7/sqtwolog/soft thresh. combinations achieved the similar results.

Phantom imaging studies, considering the initial SNR values in Table 3, can be divided into five different groups as; very low level noise (SNR $\geq 100)$, low level noise (100 $\geq$ SNR $\geq 70)$, medium level noise $(70>$ SNR $\geq 40)$, high level noise $(40>$ SNR $\geq 10)$ and very high level noise $(10>$ SNR). Fig. 6 and 7 shows the example images generated using raw signal and filtered signals. 


\subsection{Blood Cell Study Results}

In this section, PA signals acquired from the PAM system for the imaging of blood cells were denoised. In the process, the same procedure was applied as in the first and second sections. At this study, an object which has certain boundaries as in phantom imaging study was not found. Therefore, for the evaluation of the filter performance, standard deviation values in the background are calculated. Table 4 shows the calculated standard deviation values for different filtering approaches.

Table 4 shows that sym7/sqtwolog/soft thresh. combination achieved the best improvement. Results of the LPF and BPF were very close to each other, however, they showed lower performance compared to sym7/sqtwolog/soft thresh. combination. Fig. 8 shows the example images generated using raw and filtered signal (using sym7/sqtwolog/soft thresh. combination) for blood cell imaging study.

\section{DISCUSSION}

In denoising process, simulation studies were planned as preliminary work of other two steps. In this step, simulated PA signals were used. Table 1 shows that in general, bior3.5 and bior3.7 wavelets gave similar results whereas sym7 wavelet outperformed both, for all noise levels. For LPF and BPF cases, as the noise level increased their performance got worse. Using this performance comparison, LPF, BPF and bior3.7 and sym7 wavelets (to perform DWT based filtering) were chosen for the next steps.

For a realistic PAM systems, acoustic detectors also generate their own noise [12]. In this study, PA signal was simulated only considering PA source. For simulation studies, 
the effect of the acoustic detector on simulated signal was not modelled; however, the effect of the detector was inherently included in all experimental data.

The performance of rigrsure and heursure algorithms, which use standard deviation in calculations, vary with the level of noise in the signal. In images with low initial SNR value the standard deviation is usually high. For the phantom study, in images which have low initial SNR, rigrsure and heursure algorithms achieved similar results but showed lower performance than the sqtwolog and minimaxi algorithms. For images with high initial SNR value, improvement using rigrsure and heursure algorithms approached to the sqtwolog and minimaxi algorithms. Sqtwolog algorithm uses a threshold value updated at each level according to the noise coefficient (estimated from data) at that level. Thus, the performance of the algorithm depends on the noise in data. In images with high-level background noise this algorithm performed well. In images with very high-level background noise, however, amplitude loss was increased due to increased noise coefficient and performance of the algorithm decreased compared to the minimaxi. At this noise level, minimaxi algorithm preserved the amplitude of the PA signal and achieved better performance than sqtwolog. Sym7 wavelet adopted well to the PA signals regardless of the thresholding method chosen due to the similarity between this wavelet and the structure of PA signal. LPF and BPF, however, outperformed the performance of sym7 in cases with low/vey-low noise levels.

In blood cell study, results supported the performance results of the filters mentioned above. In the reconstructed image, standard deviation of the background was similar to 
those in I1 and I2 phantom images. For these images the best method was sym7/sqtwolog/soft thresh. combination which is consistent with the experimental phantom results.

There are few studies on filtering of PA signals in literature. In one of them, Holan and Viator [18] used a laser light source with wavelength of $450 \mathrm{~nm}, 543 \mathrm{~nm}$ and a laser with pulse energy ranging from 1.3 to $3.5 \mathrm{~mJ}$ to generate PA signals. For filtering purpose, they used a wavelet transform based (level independent sqtwolog/soft thresh.) algorithm and achieved $22 \%$ improvement in the blood vessel images they reconstructed using recorded PA signals. The results show that our approach achieves better improvement for a variety of cases. In other studies, Hill et al. [11] investigated the reduction of laser-induced noise by singular value decomposition method and Kong et al. [24] used an approach, based on empirical mode decomposition for denoising of photoacoustic signals in frequency domain. In these studies, improvement was not numerically evaluated, thus it was not possible to make a comparison.

In this work, denoising of the test target signals and blood cell imaging signals from the PAM system were studied and discussed in a systematic way. In literature, vascular PA imaging has been examined by many researchers [25]-[28]. In future works, concept of this study can be extended on denoising and analyzing of PA signals for vascular imaging and other common imaging studies.

\section{CONCLUSIONS}


In this study, we have proposed an image reconstruction algorithm that implements different filtering approaches for denoising photoacoustic signals. The study was carried out in three steps. In the first step, simulated PA signals were filtered and appropriate mother wavelets were selected as bior3.7 and sym7 among a set of mother wavelets. In the second step, experimental data collected for eleven different cases were used. Results show that at different noise levels, different methods are effective for SNR and CNR improvements. Methods having similar SNR improvement were evaluated according to their performance in CNR improvement. For very low level noise, we only observed an improvement in SNR, whereas CNR almost remained the same. Effective methods for SNR and CNR improvement were determined as follows;

- SNR $\geq 100$ (very low level noise) : Band-pass filter (only SNR improvement)

- $\quad 100 \geq \mathrm{SNR} \geq 70$ (low level noise) : Low-pass filter

- $70>$ SNR $\geq 10$ (medium and high level noise): sym7/sqtwolog/soft thresh.

- 10> SNR (Very high level noise): sym7/minimaxi/soft thresh.

In the last step, blood cell signals were studied and the standard deviation in the background was utilized as the comparison metric. Sym7/sqtwolog/soft thresh. combination provided the best improvement and this result is consistent with the ones obtained in experimental phantom studies. 


\section{Funding}

This study was financially supported by the Ankara University Scientific Research and Projects Office (Grant No: 17L0443010) and the Republic of Turkey Ministry of Development (Grant No: 2009 K120520).

\section{Author Contributions}

This study is the Master Thesis of G.G. under guidance of O.B.'s supervision. The experimental work carried out in M.B.U.'s Medical and Biological Physics Research Group laboratory. Experimental system set up and data collection were done by N.U., A.D. and E.A.K.

\section{References}

[1]. L. V. Wang, "Tutorial on photoacoustic microscopy and computed tomography," IEEE J. Sel. Top. Quantum Electron., vol. 14, no. 1, pp. 171-179, 2008.

[2]. L. V Wang, "Prospects of photoacoustic tomography.," Med. Phys., vol. 35, no. 12, pp. $5758-67,2008$.

[3] L. Changhui, and L.V. Wang. "Photoacoustic tomography and sensing in biomedicine." Physics in Medicine \& Biology 54.19 (2009): R59.

[4] Kolkman, R. G., Thumma, K. K., Gerbert, A. T., Siphanto, R. I., van Neck, H., Steenbergen, W., \& van Leeuwen, T. G. (2008, February). Photoacoustic imaging of tumor angiogenesis. In Photons Plus Ultrasound: Imaging and Sensing 2008: The Ninth Conference on Biomedical Thermoacoustics, Optoacoustics, and Acousto-optics (Vol. 6856, p. 685602). International Society for Optics and Photonics.

[5] Jansen, K., Van Der Steen, A. F., van Beusekom, H. M., Oosterhuis, J. W., \& van Soest, G. (2011). Intravascular photoacoustic imaging of human coronary atherosclerosis. Optics letters, 36(5), 597-599. 
[6] Papadopoulos, I. N., Simandoux, O., Farahi, S., Pierre Huignard, J., Bossy, E., Psaltis, D., \& Moser, C. (2013). Optical-resolution photoacoustic microscopy by use of a multimode fiber. Applied Physics Letters, 102(21), 211106.

[7]Allen, T. J., Ogunlade, O., Zhang, E., \& Beard, P. C. (2018). Large area laser scanning optical resolution photoacoustic microscopy using a fibre optic sensor. Biomedical optics express, 9(2), 650-660.

[8] Wang, Lihong V., Photoacoustic imaging and spectroscopy. CRC press, 2017.

[9] Yao, J., \& Wang, L. V. (2014). Sensitivity of photoacoustic microscopy. Photoacoustics, 2(2), 87-101.

[10] Cheng, J. (2017). Investigating signal denoising and iterative reconstruction algorithms in photoacoustic tomography (T). University of British Columbia. From,https://open.library.ubc.ca/cIRcle/collections/24/items/1.0354460

[11] Hill, Emma R., et al. "Identification and removal of laser-induced noise in photoacoustic imaging using singular value decomposition." Biomedical optics express 8.1 (2017): 68-77.

[12] Winkler, A. M., Maslov, K. I., \& Wang, L. V. (2013). Noise-equivalent sensitivity of photoacoustics. Journal of biomedical optics, 18(9), 097003.

[13] Hansen, M., and Yu, B. (2000). Wavelet thresholding via MDL for natural images. IEEE Transactions on Information Theory, 46(5), 1778-1788. [11] Donoho, David L. "De- noising by soft-thresholding." IEEE transactions on information theory 41.3 (1995): $613-627$.

[14] Alfaouri, Mikhled, and Khaled Daqrouq. "ECG signal denoising by wavelet transform thresholding." American Journal of applied sciences 5.3 (2008): 276281.

[15] Aqil, Mounaim, Atman Jbari, and Abdennasser Bourouhou. "ECG Signal Denoising by Discrete Wavelet Transform." International Journal of Online Engineering (iJOE) 13.09 (2017): 51-68.

[16] Veer, Karan, and Ravinder Agarwal. "Wavelet denoising and evaluation of electromyogram signal using statistical algorithm." International Journal of Biomedical Engineering and Technology16.4 (2014): 293-305. 
[17] Gradolewski, Dawid, et al. "Arm EMG wavelet-based denoising system." Mechatronics-Ideas for Industrial Application. Springer, Cham, 2015. 289-296.

[18] Holan, Scott H., and John A. Viator. "Automated wavelet denoising of photoacoustic signals for circulating melanoma cell detection and burn image reconstruction." Physics in Medicine \& Biology 53.12 (2008): N227.

[19] Erkol, Hakan, Esra Aytac-Kipergil, and Mehmet Burcin Unlu. "Photoacoustic radiation force on a microbubble." Physical Review E 90.2 (2014): 023001.

[20] Aytac-Kipergil, Esra, et al. "Development of a fiber laser with independently adjustable properties for optical resolution photoacoustic microscopy." Scientific reports 6 (2016): 38674.

[21] Ngui, Wai Keng, et al. "Wavelet analysis: mother wavelet selection methods." Applied mechanics and materials. Vol. 393. Trans Tech Publications, 2013.

[22] Patil, Pradnya B., and Mahesh S. Chavan. "A wavelet based method for denoising of biomedical signal." Pattern Recognition, Informatics and Medical Engineering (PRIME), 2012 International Conference on. IEEE, 2012.

[23] Donoho, David L., and Jain M. Johnstone. "Ideal spatial adaptation by wavelet shrinkage." biometrika 81.3 (1994): 425-455.

[24] Kong, Qinglin, et al. "Denoising signals for photoacoustic imaging in frequency domain based on empirical mode decomposition." Optik 160 (2018): 402-414.

[25] Rao, B., Li, L., Maslov, K., \& Wang, L. (2010). Hybrid-scanning optical-resolution photoacoustic microscopy for in vivo vasculature imaging. Optics letters, 35(10), 1521-1523.

[26] Liu, W., Schultz, K. M., Zhang, K., Sasman, A., Gao, F., Kume, T., \& Zhang, H. F. (2014). In vivo corneal neovascularization imaging by optical-resolution photoacoustic microscopy. Photoacoustics, 2(2), 81-86.

[27] Jeon, S., Song, H. B., Kim, J., Lee, B. J., Managuli, R., Kim, J. H., \& Kim, C. (2017). In vivo photoacoustic imaging of anterior ocular vasculature: a random sample consensus approach. Scientific reports, 7(1), 4318. 
[28] Zhao, H., Wang, G., Lin, R., Gong, X., Song, L., Li, T., \& Li, L. (2018). Threedimensional Hessian matrix-based quantitative vascular imaging of rat iris with optical-resolution photoacoustic microscopy in vivo. Journal of biomedical optics, 23(4), 046006.

[29] https://www.thorlabs.com/newgrouppage9.cfm?objectgroup_id=4338 


\begin{tabular}{|c|c|c|c|c|c|c|c|}
\hline & & \multicolumn{2}{|c|}{ Low Noise } & \multicolumn{2}{|c|}{ Medium Noise } & \multicolumn{2}{|c|}{ High Noise } \\
\hline & None & \multicolumn{2}{|c|}{0.30} & \multicolumn{2}{|c|}{0.50} & \multicolumn{2}{|c|}{1.00} \\
\hline & LPF & \multicolumn{2}{|c|}{0.13} & \multicolumn{2}{|c|}{0.20} & \multicolumn{2}{|c|}{0.40} \\
\hline \multicolumn{2}{|r|}{ BPF } & \multicolumn{2}{|c|}{0.13} & \multicolumn{2}{|c|}{0.21} & \multicolumn{2}{|c|}{0.42} \\
\hline & & Soft & Hard & Soft & Hard & Soft & Hard \\
\hline \multirow{4}{*}{ 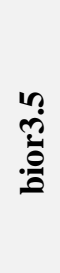 } & Sqtwolog & 0.13 & 0.19 & 0.18 & 0.34 & 0.31 & 0.65 \\
\hline & Rigrsure & 0.20 & 0.28 & 0.34 & 0.47 & 0.69 & 0.95 \\
\hline & Heursure & 0.19 & 0.22 & 0.32 & 0.36 & 0.64 & 0.73 \\
\hline & Minimaxi & 0.14 & 0.26 & 0.22 & 0.43 & 0.41 & 0.86 \\
\hline \multirow{4}{*}{ مُ } & Sqtwolog & 0.13 & 0.15 & 0.18 & 0.27 & 0.30 & 0.52 \\
\hline & Rigrsure & 0.19 & 0.28 & 0.32 & 0.47 & 0.63 & 0.94 \\
\hline & Heursure & 0.18 & 0.21 & 0.29 & 0.36 & 0.58 & 0.71 \\
\hline & Minimaxi & 0.12 & 0.23 & 0.20 & 0.38 & 0.36 & 0.77 \\
\hline \multirow{4}{*}{ 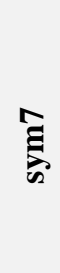 } & Sqtwolog & 0.14 & 0.13 & 0.19 & 0.18 & 0.31 & 0.31 \\
\hline & Rigrsure & 0.13 & 0.21 & 0.19 & 0.34 & 0.37 & 0.69 \\
\hline & Heursure & 0.14 & 0.11 & 0.19 & 0.17 & 0.31 & 0.31 \\
\hline & Minimaxi & 0.13 & 0.18 & 0.18 & 0.29 & 0.30 & 0.55 \\
\hline
\end{tabular}

Table 1. Calculated RMSE values for Gaussian noise 


\begin{tabular}{|c|c|c|c|}
\hline $\begin{array}{c}\text { Study } \\
\text { Number }\end{array}$ & $\begin{array}{c}\text { Wavelength } \\
(\mathbf{n m})\end{array}$ & $\begin{array}{c}\text { Laser Power } \\
(\mathbf{m W})\end{array}$ & $\begin{array}{c}\text { Pulse Energy } \\
(\boldsymbol{\mu} \mathbf{J})\end{array}$ \\
\hline $\mathbf{I 1}$ & 785 & 32 & 0.49 \\
\hline $\mathbf{I 2}$ & 785 & 32 & 0.49 \\
\hline $\mathbf{I 3}$ & 785 & 32 & 0.49 \\
\hline $\mathbf{I 4}$ & 785 & 34 & 0.52 \\
\hline $\mathbf{I 5}$ & 697 & 33 & 0.51 \\
\hline $\mathbf{I 6}$ & 880 & 33 & 0.51 \\
\hline $\mathbf{I 7}$ & 732 & 28 & 0.43 \\
\hline $\mathbf{I 8}$ & 650 & 34 & 0.52 \\
\hline $\mathbf{I 9}$ & 785 & 35 & 0.54 \\
\hline $\mathbf{I 1 0}$ & 880 & 32 & 0.49 \\
\hline $\mathbf{I 1 1}$ & 697 & 34 & 0.52 \\
\hline
\end{tabular}

Table 2. System parameters used in phantom imaging 


\begin{tabular}{|c|c|c|c|c|c|c|}
\hline $\begin{array}{c}\text { Study } \\
\text { Number }\end{array}$ & $\begin{array}{l}\text { Initial } \\
\text { SNR }\end{array}$ & $\begin{array}{l}\text { Initial } \\
\text { CNR }\end{array}$ & $\begin{array}{c}\text { SNR \% } \\
\text { Improvement }\end{array}$ & $\begin{array}{c}\text { CNR \% } \\
\text { Improvement }\end{array}$ & $\begin{array}{c}\text { SNR Improvement } \\
\text { Methods }\end{array}$ & $\begin{array}{c}\text { CNR Improvement } \\
\text { Methods }\end{array}$ \\
\hline \multirow{3}{*}{ I1 } & \multirow{3}{*}{36.22} & \multirow{3}{*}{11.41} & 409.47 & 61.25 & sym7/sqtwolog/soft & sym7/sqtwolog/soft \\
\hline & & & 191.09 & 59.94 & sym7/minimaxi/soft & sym7/minimaxi/soft \\
\hline & & & 159.66 & 58.24 & BGF & bior3.7/sqtwolog/soft \\
\hline \multirow{3}{*}{ I2 } & \multirow{3}{*}{26.71} & \multirow{3}{*}{11.67} & 415.14 & 41.56 & sym7/sqtwolog/soft & sym7/sqtwolog/soft \\
\hline & & & 191.65 & 35.81 & BPF & BPF \\
\hline & & & 170.11 & 32.67 & LPF & LPF \\
\hline \multirow{3}{*}{ I3 } & \multirow{3}{*}{5.28} & \multirow{3}{*}{1.56} & 142.55 & 300.75 & sym7/minimaxi/soft & sym7/minimaxi/soft \\
\hline & & & 138.45 & 298.24 & bior3.7/rigrsure/soft & bior3.7/rigrsure/soft \\
\hline & & & 134.28 & 298.15 & sym7/sqtwolog/soft & sym7/sqtwolog/soft \\
\hline \multirow{3}{*}{ I4 } & \multirow{3}{*}{8.47} & \multirow{3}{*}{3.00} & 175.47 & 228.09 & sym7/minimaxi/soft & sym7/minimaxi/soft \\
\hline & & & 151.77 & 209.44 & bior3.7/minimaxi/soft & bior3.7/minimaxi/soft \\
\hline & & & 146.29 & 205.01 & bior3.7/heursure/hard & bior3.7/heursure/hard \\
\hline \multirow{3}{*}{ I5 } & \multirow{3}{*}{45.70} & \multirow{3}{*}{6.34} & 92.10 & 15.02 & sym7/sqtwolog/soft & sym7/sqtwolog/soft \\
\hline & & & 85.20 & 11.50 & BPF & $\mathrm{BPF}$ \\
\hline & & & 81.79 & 11.40 & LPF & LPF \\
\hline \multirow{3}{*}{ I6 } & \multirow{3}{*}{7.80} & \multirow{3}{*}{3.54} & 163.95 & 156.30 & sym7/minimaxi/soft & sym7/minimaxi/soft \\
\hline & & & 121.59 & 153.14 & bior3.7/minimaxi/soft & bior3.7/rigrsure/soft \\
\hline & & & 116.53 & 152.49 & sym7/rigrsure/soft & bior3.7/rigrsure/soft \\
\hline \multirow{3}{*}{ I7 } & \multirow{3}{*}{54.143} & \multirow{3}{*}{16.28} & 159.39 & 12.45 & sym7/sqtwolog/soft & sym7/sqtwolog/soft \\
\hline & & & 61.56 & 11.56 & BPF & bior3.7/rigrsure/soft \\
\hline & & & 59.45 & 11.19 & LPF & bior3.7/rigrsure/soft \\
\hline \multirow{3}{*}{ I8 } & \multirow{3}{*}{105.60} & \multirow{3}{*}{43.22} & 61.77 & -8.60 & BPF & bior3.7/sqtwolog/soft \\
\hline & & & 60.37 & -9.65 & LPF & sym7/heursure/soft \\
\hline & & & 54.80 & -10.92 & sym7/sqtwolog/soft & bior3.7/rigrsure/soft \\
\hline \multirow{3}{*}{ I9 } & \multirow{3}{*}{71.40} & \multirow{3}{*}{22.06} & 65.61 & 15.86 & LPF & LPF \\
\hline & & & 64.37 & 11.42 & sym7/sqtwolog/soft & sym7/sqtwolog/soft \\
\hline & & & 60.18 & 9.11 & BGF & sym7/minimaxi/soft \\
\hline \multirow{3}{*}{$\mathbf{I 1 0}$} & \multirow{3}{*}{64.12} & & 63.04 & 4.88 & sym7/sqtwolog/soft & sym7/sqtwolog/soft \\
\hline & & 17.83 & 59.44 & 3.11 & BPF & sym7/rigrsure/soft \\
\hline & & & 57.63 & 2.29 & LPF & sym7/minimaxi/soft \\
\hline & & & 182.73 & 110.02 & LPF & sym7/sqtwolog/soft \\
\hline I11 & 44.94 & 17.16 & 174.30 & 100.30 & BPF & sym7/minimaxi/soft \\
\hline & & & 150.44 & 92.33 & sym7/sqtwolog/soft & bior3.7/minimaxi/soft \\
\hline
\end{tabular}

Table 3. Phantom images SNR and CNR improvements 


\begin{tabular}{|c|c|c|c|}
\hline Filter & ---- & \multicolumn{2}{|c|}{ Std } \\
\hline None & ---- & \multicolumn{2}{|c|}{0.048} \\
\hline L.P & ---- & \multicolumn{2}{|c|}{0.044} \\
\hline B.P & ---- & \multicolumn{2}{|c|}{0.044} \\
\hline$\cdots$ & Threshold & Soft & Hard \\
\hline \multirow{4}{*}{ ஜூٌّ } & Sqtwolog & 0.018 & 0.027 \\
\hline & Rigrsure & 0.042 & 0.058 \\
\hline & Heursure & 0.019 & 0.044 \\
\hline & Minimaxi & 0.027 & 0.062 \\
\hline \multirow{4}{*}{ 気 } & Sqtwolog & 0.012 & 0.015 \\
\hline & Rigrsure & 0.030 & 0.056 \\
\hline & Heursure & 0.014 & 0.032 \\
\hline & Minimaxi & 0.017 & 0.051 \\
\hline
\end{tabular}

Table 4. Blood cell images background standard deviations 

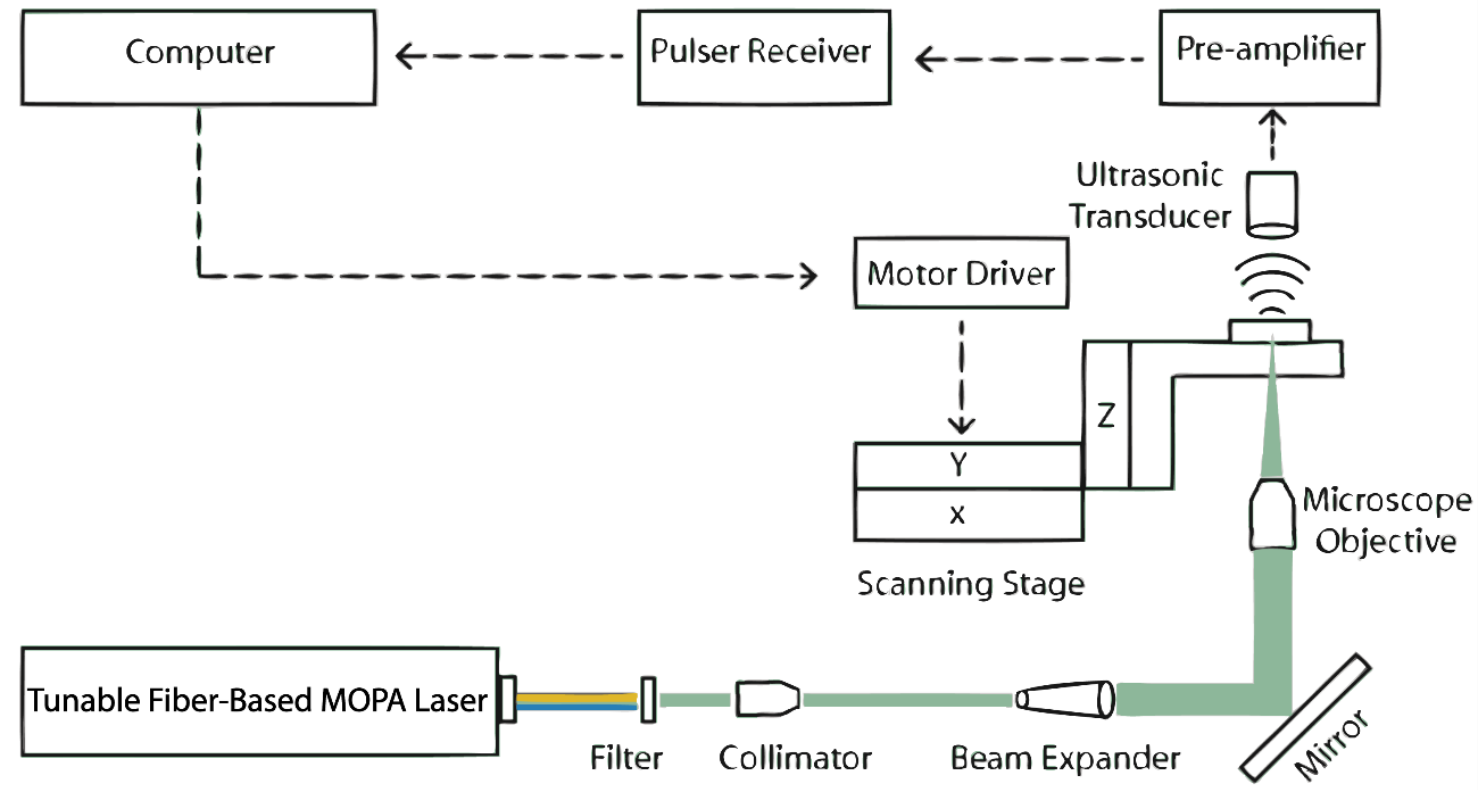

Figure 1. Main blocks of PAM system [20] 


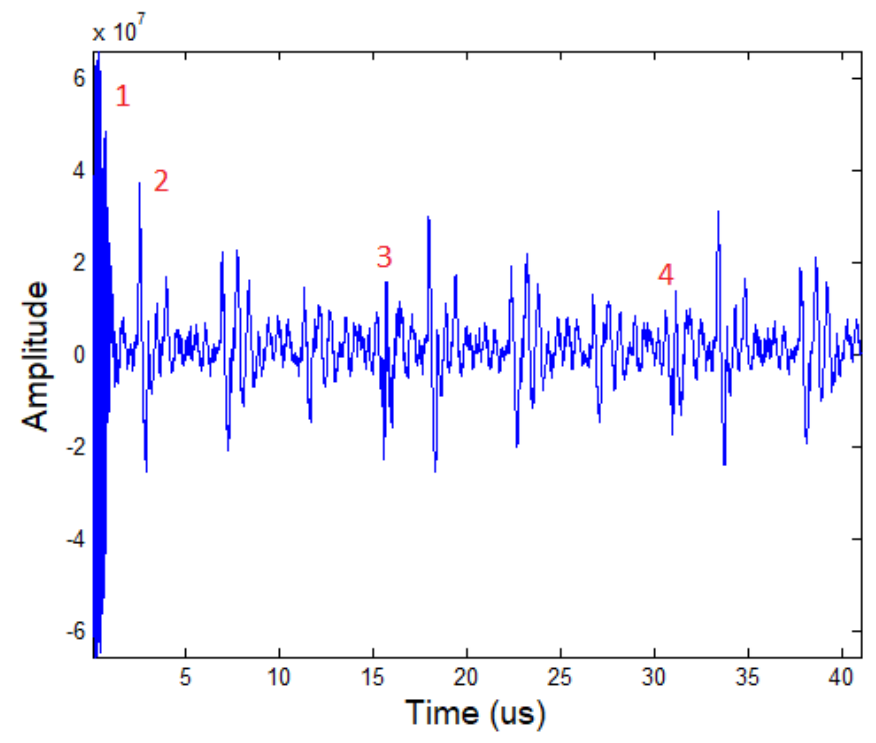

Figure 2. Typical PA signal (1, 3 and 4 show the triggering signal and 2 shows the PA signal) 


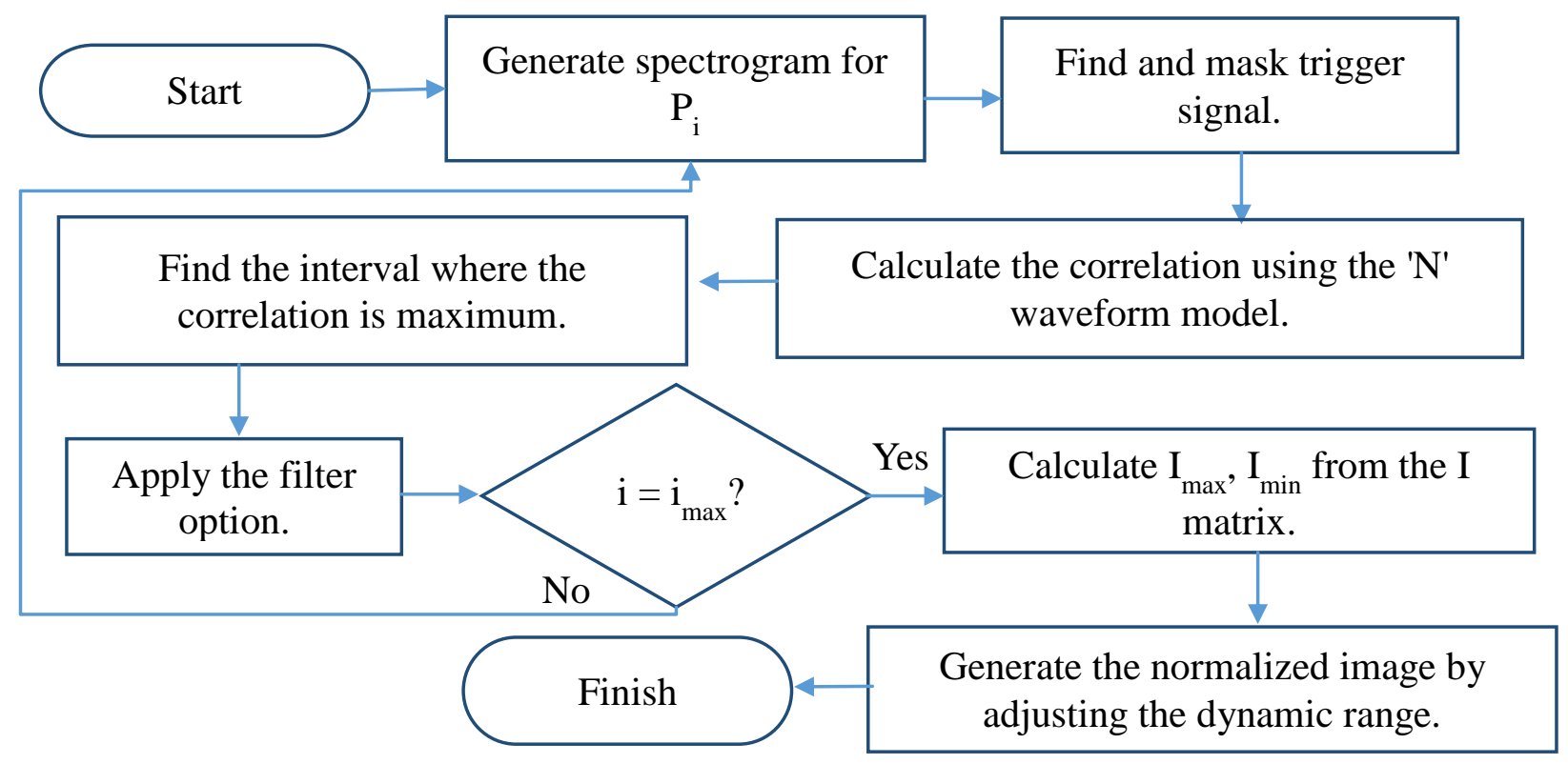

Figure 3. Image reconstruction algorithm 


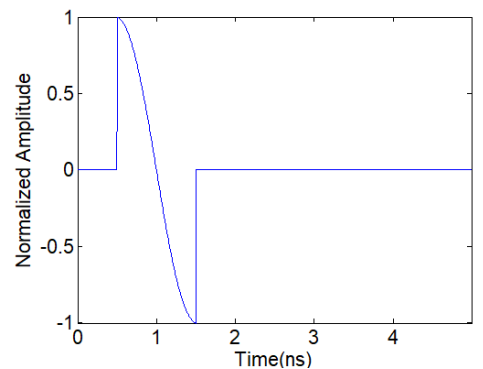

(a)

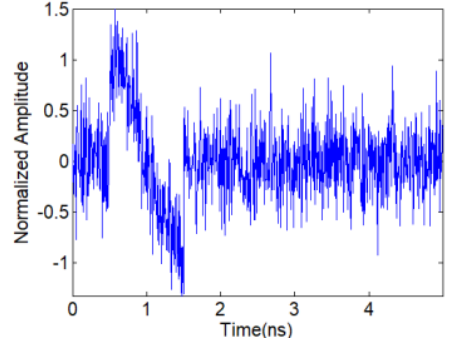

(b)

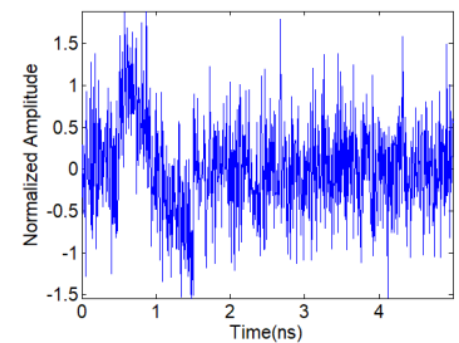

(e)

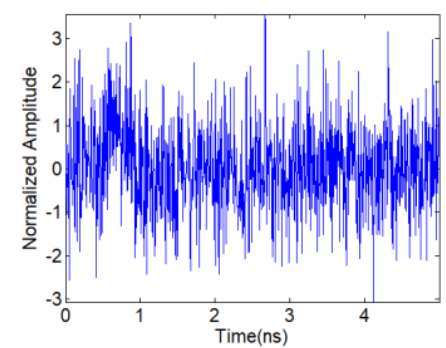

(h)

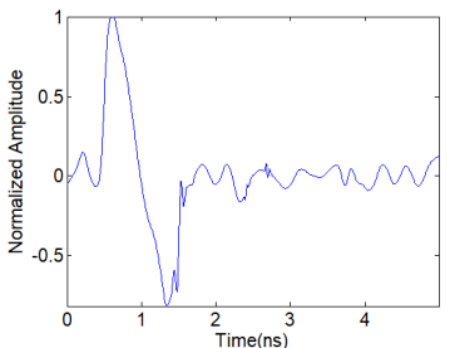

(c)

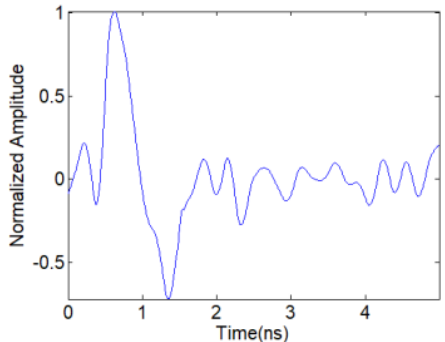

(f)

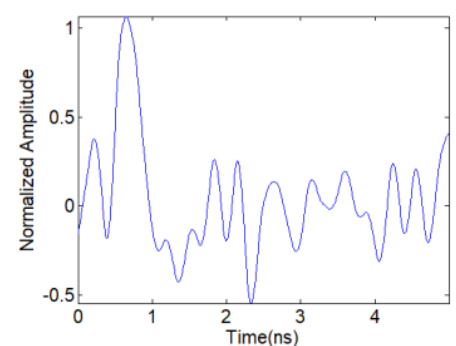

(i)

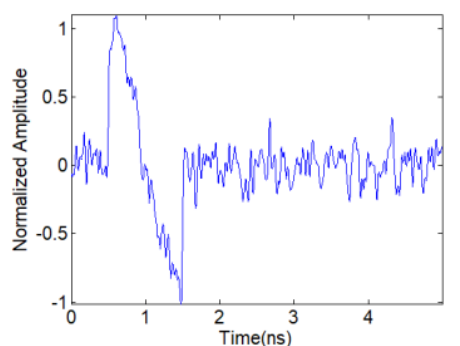

(d)

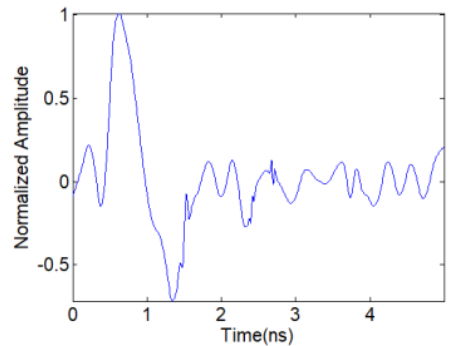

$(\mathrm{g})$

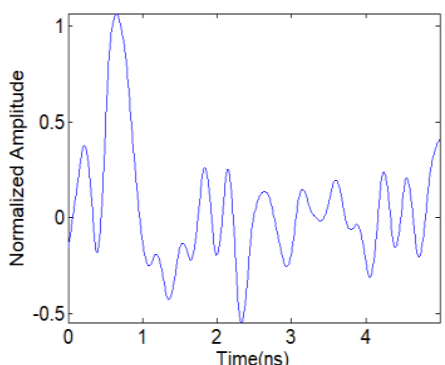

(j)

Figure 4. Simulated signal, noise added signals and filtered signal examples (a) simulated signal, (b) 30\% noisy signal, (c) 30\% noisy - sym7/heursure/hard thresh, (d) $30 \%$ noisy - LPF, (e) $50 \%$ noisy signal (f) $50 \%$ noisy - sym7/heursure/hard thresh., (g) $50 \%$ noisy sym7/minimaxi/soft thresh., (h) $100 \%$ noisy signal, (i) $100 \%$ noisy bior3.7/sqtwolog/soft thresh., (j) 100\% noisy - sym7/minimaxi/soft thresh. 


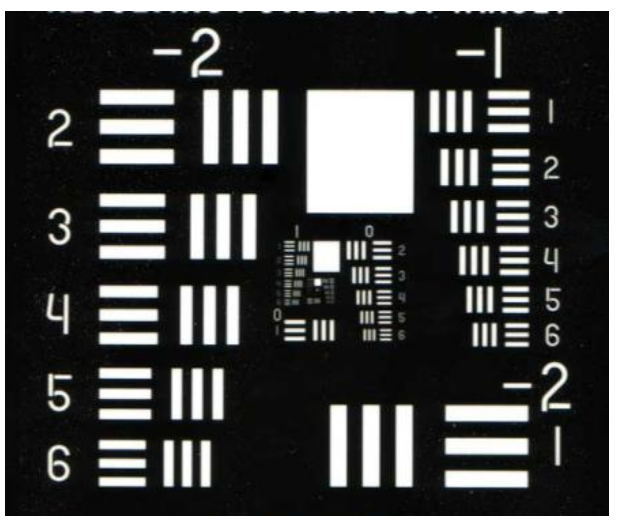

Figure 5. USAF Resolution Test Target [29] 


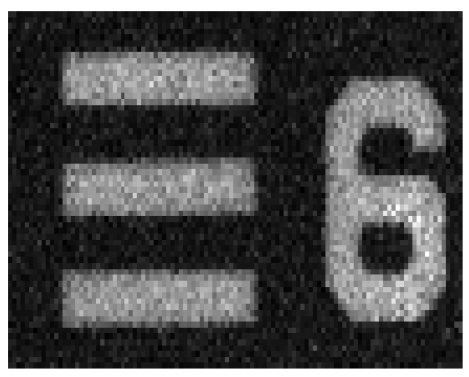

(a)

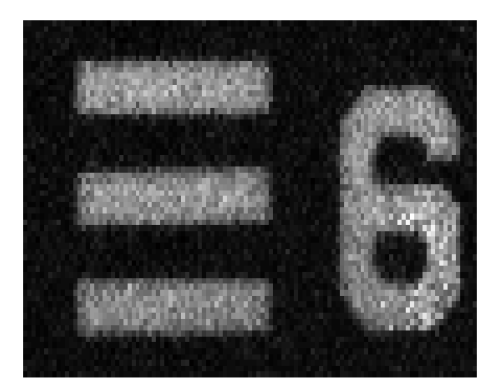

(d)

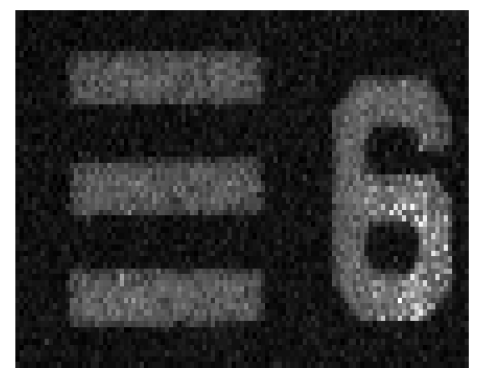

(b)

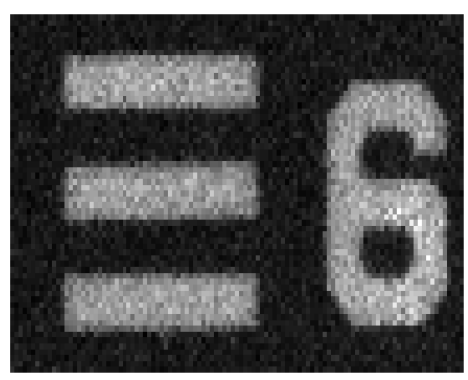

(e)

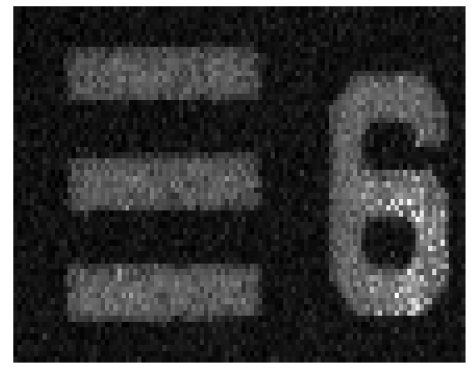

(c)

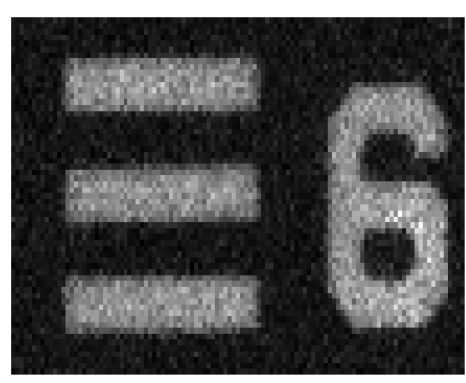

(f)

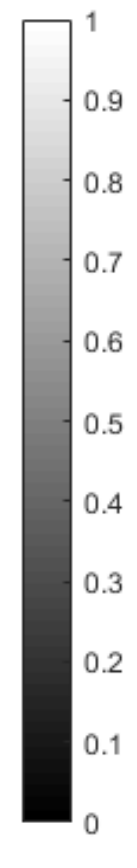

f)

Figure 6. I6 imaging study (image matrix size $=61 \times 109$, pixel size $=0.001 \mu \mathrm{m}$ ) (a) raw image, (b) LPF, (c) BPF, (d) sym7/sqtwolog/soft thresh., (e) sym7/minimaxi/soft thresh., (f) bior3.7/minimaxi/soft thresh. 


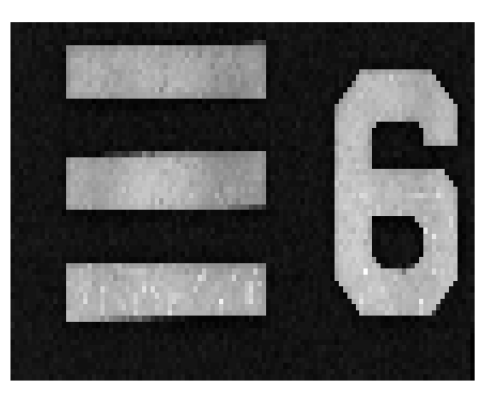

(a)

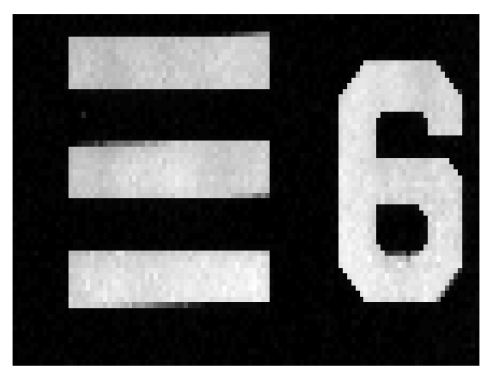

(d)

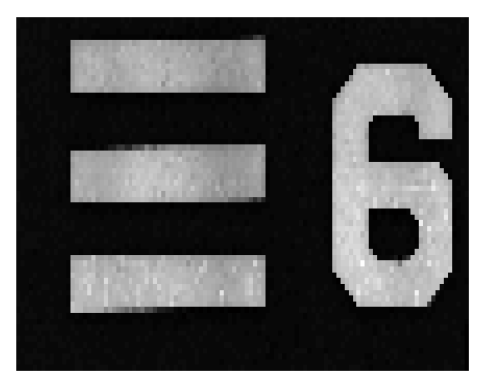

(b)

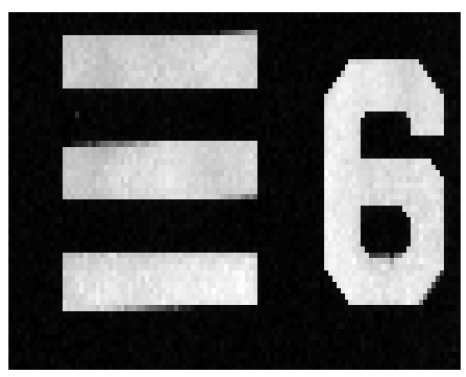

(e)

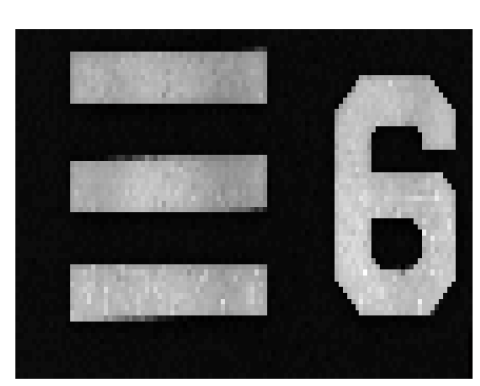

(c)

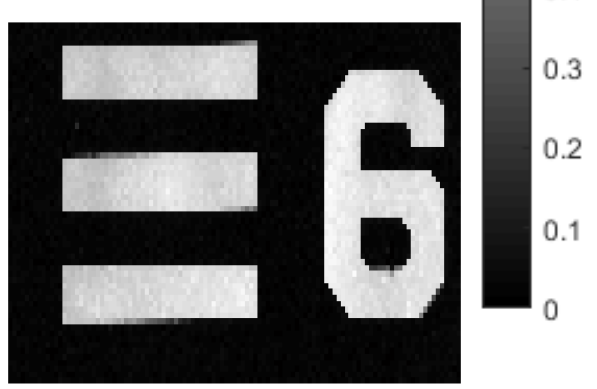

(f)

Figure 7. I1 imaging study (image matrix size $=61 \times 109$, pixel size $=0.001 \mu \mathrm{m}$ )

(a) raw image, (b) LPF, (c) BPF, (d) sym7/sqtwolog/soft thresh., (e) sym7/minimaxi/soft thresh., (f) bior3.7/minimaxi/soft thresh. 


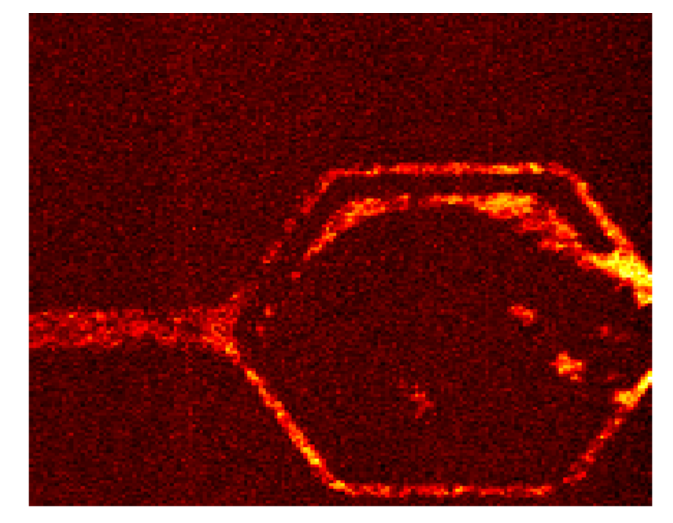

(a)

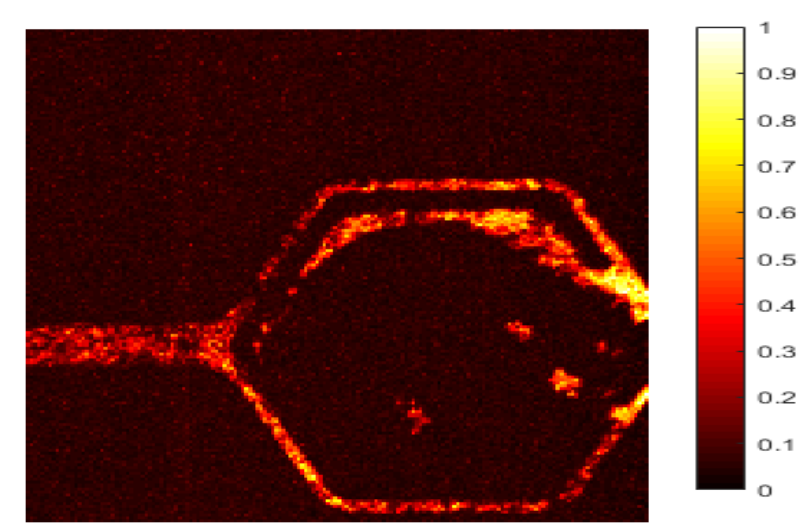

(b)

Figure 8. Blood cell imaging study (image matrix size $=168 \times 168$, pixel size $=0.001$ $\mu \mathrm{m}$ ) (a) original image (using raw signal), (b) sym7/sqtwolog/soft thresh. image 